\title{
Partial Models: Towards Modeling and Reasoning with Uncertainty
}

\author{
Michalis Famelis, Rick Salay, and Marsha Chechik \\ University of Toronto, Canada
}

\begin{abstract}
Models are good at expressing information about software but not as good at expressing modelers' uncertainty about it. The highly incremental and iterative nature of software development nonetheless requires the ability to express uncertainty and reason with models containing it. In this paper, we build on our earlier work on expressing uncertainty using partial models, by elaborating an approach to reasoning with such models. We evaluate our approach by experimentally comparing it to traditional strategies for dealing with uncertainty as well as by conducting a case study using open source software. We conclude that we are able to reap the benefits of well-managed uncertainty while incurring minimal additional cost.
\end{abstract}

\section{INTRODUCTION}

Software engineering is a highly incremental and iterative endeavor where uncertainty can exist at multiple stages of the development process. Consequently, systematic approaches to handling uncertainty are essential throughout the software life-cycle.

Models are used pervasively in software engineering, and their ability to express information about different aspects of software has been studied by many researchers [25]. However, models seldom provide the means for expressing the uncertainty that the modeler has about this information. In this paper, by "uncertainty" we mean "multiple possibilities". This notion of uncertainty is often used in behavioral modeling [10], but we expand it to arbitrary modeling languages. For example, a modeler of a class diagram may be uncertain about which of two attributes to include in a particular class because they represent different design strategies, and it is too early to know which is correct.

Uncertainty can be introduced into the modeling process in many ways: alternative ways to fix model inconsistencies [14], [5], [24], different design alternatives (e.g., the above example) [26], problem-domain uncertainties [27], multiple stakeholder opinions [18], etc. In each case, the presence of uncertainty means that, rather than having a single model, we actually have a set of possible models and we are not sure which is the correct one. Living with uncertainty requires us to keep track of this set and use it within modeling activities wherever we would use an individual model; however, this can be challenging since modeling activities are typically intended for individual models, not sets of them. Also, managing a set of models explicitly is impractical as its size might be quite large. E.g., in Sec. VI we give a case study in which two inconsistencies lead to several hundred possible models. On the other hand, if uncertainty is ignored and one particular possible model is chosen prematurely, we risk having incorrect information in the model.
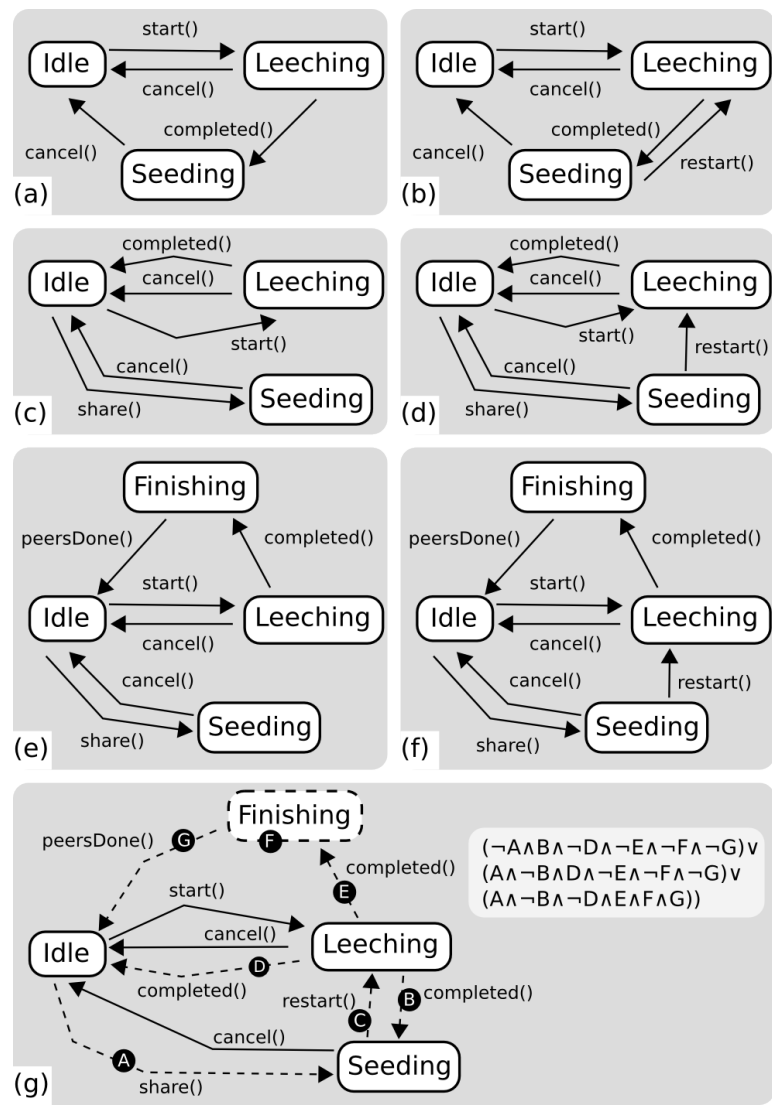

Figure 1. (a-f) Six alternative designs for a peer-to-peer file sharing system; (g) a partial model $M_{e}$ for the six alternatives.

Our approach to handling uncertainty is to use annotations with well-defined semantics that change a model into a partial model, i.e., one that compactly yet precisely encodes the entire set of possible models. This representation allows us to work with a set of models as if it were a single model and do reasoning efficiently with all the possible models simultaneously.

Motivating Example. To help motivate and explain our approach, we use the example of a team engaged in the development of a simple peer-to-peer file sharing application. The team uses UML State Machine diagrams to model the behavior of this application. Its states are Idle, Leeching (downloading a file) and seeding (sharing a complete local copy). Downloading always starts from the Idle state, and Seeding and Leeching can always be canceled. We assume that at this stage of development, the team has not finalized 
the exact behavior of the program, due to vague requirements given to them by their client. The team has drafted three alternative behavioral designs:

1) "Benevolent": Once the file is downloaded, the program automatically starts Seeding, as shown in Fig. 1(a).

2) "Selfish": Once the file is downloaded, the program becomes Idle, and the user can choose whether to start Seeding or not - see Fig. 1(c).

3) "Compromise": Once the file is downloaded, the program stops accepting new peers. It doesn't disconnect from peers that were already connected during the Leeching stage, but rather waits while they are Finishing before it becomes Idle - see Fig. 1(e).

The team is also unsure whether the program should allow the user to restart a finished download (i.e., download the file again). The three alternatives with this feature are shown in Fig. 1(b, d, e), respectively.

Until the client clarifies the requirements, the team is faced with uncertainty over which design decision to choose. At this point, it is probably useful to be able to reason about the available choices, both to ensure that the models conform to the desired constraints and to explore their properties. For example, assume that the team is using a code generator that, in order to ensure determinism, requires two hard constraints: HC1: No two transitions have the same source and target. HC2: No state is a sink.

Additionally, the team is interested in two "nice-to-have" properties, i.e., soft constraints that are not strictly required but are desirable:

SC1: Users can share files they already have, (i.e., Seeding is directly reachable from Idle).

SC2: Users can always cancel any operation (i.e., every nonidle state has a transition to Idle on cancel()).

In order to reason effectively about any of these properties over the entire set of alternatives, the team may want to ask the following questions:

Does the property hold for all, some or none of the alternatives? This can help determine how critical some property is in selecting alternatives when uncertainty is lifted. For example, HC2 holds for all alternatives, and therefore is not going to be a main reason in selecting one, once uncertainty is resolved. Moreover, if some property does not hold for any alternative, it may be an indication that the team needs to revisit the designs, sooner rather than later. For example, knowing early on that $\mathrm{HC} 1$ does not hold for the alternatives in Fig. 1(c, d) may be an indication that the team needs to reconsider the design of the "selfish" scenario.

If the property does not hold for all alternatives, why is it so? This form of diagnosis can help guide development decisions even before uncertainty is lifted. Developers may be interested in finding one counter-example of an alternative where the property gets violated (or if they expected that the property would be violated - an example where it holds) to help them debug the set of alternatives. For example, locating the alternative in Fig. 1(e) might be sufficient for the team to understand why SC2 does not hold for all alternatives.

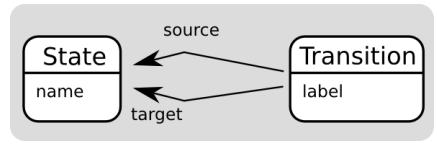

Figure 2. Simplified metamodel used for defining State Machines.

In other cases, we may prefer to calculate the entire subset of alternatives that violate the property, to explore whether there is a common underlying cause, as with the alternatives in Fig. 1(c, d) that violate the hard constraint $\mathrm{HC} 1$.

If the property is a necessary constraint, how to filter out the alternatives for which it gets violated? Developers may be interested in this sort of property-driven refinement of the set of alternatives. E.g., if the team decides that SC2 is necessary, they should be able to restrict their working set of alternatives to those that satisfy it, i.e., those in Fig. 1(a-d).

Contributions. In this paper, we elaborate and evaluate a key component of our broad research agenda for managing uncertainty within models [7]: reasoning with models containing uncertainty. Specifically, we define partial models, show how to construct them and then describe the three reasoning operators aimed to answer the questions posed by the motivating example. We then extensively evaluate our approach by experimentally comparing it to conventional strategies for dealing with uncertainty as well as by conducting a case study of an open source software project.

Organization of the rest of the paper. In Sec. II, we provide the necessary background. In Sec. III, we formally define partial models. Sec. IV develops the core methods of reasoning with partial models. These are experimentally evaluated in Sec. V and then applied to a case study in Sec. VI. We discuss related work in Sec. VII and conclude the paper with a summary and suggestions for further research in Sec. VIII.

\section{BACKGROUND}

In this section, we establish the notation and introduce concepts used in the remainder of the paper. Specifically, we ground our approach to graph-based modeling languages and propositional logic.

Modeling Formalisms. In this paper, a model is a typed graph that conforms to some metamodel represented by a distinguished type graph. Our approach is domain-independent, in the sense that it can handle arbitrary graph-based modeling languages. The definitions that follow are based on [6].

Definition 1: A graph is a tuple $G=\langle V, E, s, t\rangle$, where $V$ is a set of nodes, $E$ is a set of edges, and $s, t: E \rightarrow V$ are the source and target functions, respectively, that assign each edge a source and target node.

Definition 2: A typed graph (model) of type $T$ is a triple $\langle G$, type, $T\rangle$ consisting of a graph $G$, a metamodel $T$ and a typing function type : $G \rightarrow T$ that assigns types to the elements of $G$.

For example, the models shown in Fig. 1 are typed with the metamodel shown in Fig. 2. 
Definition 3: The scope (or vocabulary) of a model $\langle G=$ $\langle V, E, s, t\rangle$, type, $T\rangle$ is the set $S=V \cup E$ of its typed nodes and edges.

For example, the scope of the model in Fig. 1(a) consists of the states Idle, Leeching and Seeding and the edges start (), completed(), etc.

In the following, we often refer to nodes and edges that are in the scope of a model as elements or atoms of the model.

From models to formulas and back. To encode a model in propositional logic, we first map elements in its scope into propositional variables and then conjoin them. To ensure that this operation is reversible, we define specific naming conventions for the propositional variables:

- A node element $N$ of type $T$ is mapped to a propositional variable "N_T".

- An edge element $E$ of type $T$ with source node $N_{1}$ and target node $N_{2}$ is mapped to a propositional variable "E_N $\mathrm{N}_{1} \_\mathrm{N}_{2} \_\mathrm{T}$ ".

E.g., the propositional encoding of the model in Fig. 1(b) is:

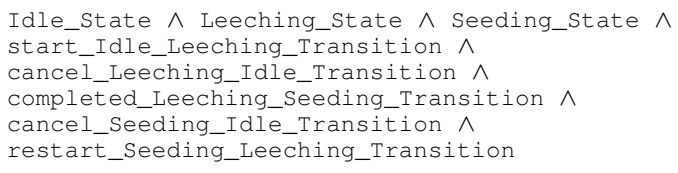

Given a propositional encoding $P(m)$, of a model $m$, we can uniquely reconstruct the model $m$ using the naming conventions. First, for every propositional whose name fits the pattern N_T, we create a node of type $T$, named $N$. Then, for every propositional variable whose name follows the pattern $\mathrm{E}_{-} \mathrm{N}_{1} \mathrm{~N}_{2}-\mathrm{T}$, we create an edge of type $T$ between the nodes $N_{1}$ and $N_{2}$, with the label $E$.

This propositional encoding also allows us to embed models into larger scopes, by negating all the variables not in the original scope. For example, the model in Fig. 1(a) can be expressed in the scope of the model in Fig. 1(b) as:

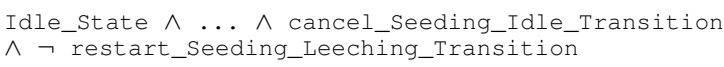

Using the propositional representation, we also define a simple form of model union. Assuming two elements with the same name are considered identical, the union of two models is a model that corresponds to a formula that is a conjunction of all the variables in the union of their scopes. For example, the union of the models in Fig. 1(b, d) is:

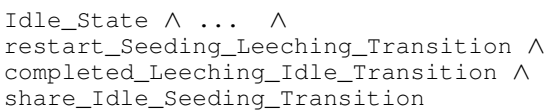

A useful extended scope is the embedding of a sparse graph into the scope of its corresponding complete graph. In the union of models with extended scopes, variables only appear negated if they are negated in both input models.

Properties. We consider properties expressed in first order logic (FOL) or in a similar language such as the Object Constraint Language (OCL) [15]. For example, the property $\mathrm{HC} 1$ is expressed in FOL as:

$$
\begin{array}{ll}
\forall t_{1}, t_{2}: \text { Transition } \quad & \left(\operatorname{Source}\left(t_{1}\right)=\operatorname{Source}\left(t_{2}\right) \wedge\right. \\
& \left.\operatorname{Target}\left(t_{1}\right)=\operatorname{Target}\left(t_{2}\right)\right) \Leftrightarrow\left(t_{1}=t_{2}\right)
\end{array}
$$

An FOL formula can be grounded over the vocabulary of a particular model that is encoded in a propositional formula. E.g., grounding $\mathrm{HC} 1$ over the vocabulary of the model in Fig. 1(a), given that it contains 4 transition elements and that $\mathrm{HC} 1$ is a universal property, results in $\Phi_{H C 1}-$ a conjunction of 10 unique terms of the form $\left(S_{i}=S_{j} \wedge T_{i}=T_{j}\right) \Leftrightarrow$ $E_{i}=E_{j}$, where $E_{i, j}$ are propositional variables representing transitions, $S_{i, j}, T_{i, j}$ are variables representing their respective source and target states, and "=" signifies identity.

\section{Partial Model Preliminaries}

In this section, we formally define partial models and their associated operations. Semantically, a partial model represents a set of classical (i.e., non-partial) models.

Partial Models. The particular type of partiality we consider in this paper is the one that allows a modeler to express uncertainty as to whether particular model atoms should be present the model. The model is accompanied by a propositional formula, called may formula, which explicates allowable combinations of such atoms.

Definition 4: A Partial Model is a tuple $\langle G, v m, e m, \phi\rangle$, where $G=\langle\langle V, E, s, t\rangle$, type $\rangle$ is a complete typed graph, $v m: V \rightarrow \mathcal{B}$ and $e m: E \rightarrow \mathcal{B}$, where $\mathcal{B}$ is the set $\{$ True, False, Maybe\}, are functions for annotating atoms in $G$, and $\phi$ is a propositional may formula over the scope $S=V \cup E$, built as described in Sec. II.

In the above definition, an annotation True (False) means that the atom must (must not) be present in the model, whereas Maybe indicates uncertainty about whether the atom should be present in the model. In other words, a partial model consists of a complete typed graph whose elements are annotated with True, False or Maybe, and a may formula that describes the allowed configurations of its elements. The annotation functions are often omitted for brevity.

Model $M_{e}$ in Fig. 1(g) is an example of a partial model. The elements annotated with True, e.g., the state Idle and the transition start(), appear with solid lines, and its Maybe elements, e.g. the state Finishing, with dashed lines. The edges that are not shown (e.g. any edge between the states Finishing and Leeching) are annotated with False. $M_{e}$ is accompanied by the may formula $\phi_{e}$, shown next to it in the figure. We used capital letters as shortcuts for the full names of the propositional variables that correspond to Maybe elements. E.g., F stands for the variable Finishing_State.

Given a partial model $M$, let $\mathcal{C}(M)$ be the set of classical (or concrete) models that it represents, called concretizations. For example, $\mathcal{C}\left(M_{e}\right)$ consists of the models shown in Fig. 1(a)-(f). A partial model with an empty set of concretizations is called inconsistent. In what follows, we only assume consistent partial models.

The size of the set of concretizations reflects the modeler's degree of uncertainty. Uncertainty can be reduced by reducing the set of concretizations via refinement. A partial model is refined by changing the annotations of its elements to increase the level of certainty: Maybe elements can be assigned to True, False or Maybe; True and False annotations must remain unchanged since information about them is already 


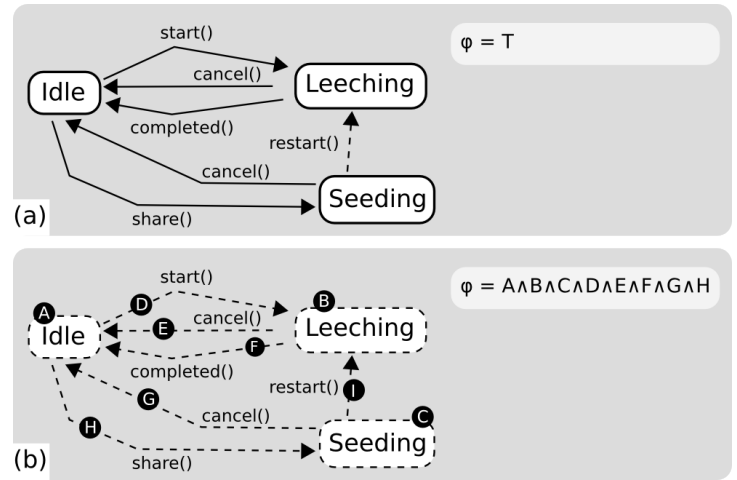

Figure 3. Normal forms of the model $M_{e}^{\neg C 1}$, shown in Fig. 4(a): (a) Graphical Normal Form (GNF) (b) Propositional Normal Form (PNF).

certain. Changes to Maybe elements must not violate the formula of the original partial model, and thus produce a (nonempty) subset of concretizations allowed by it.

Definition 5: Given two partial models $M_{1}$ and $M_{2}$, where $M_{i}=\left\langle G_{i}, v m_{i}, e m_{i}, \phi_{i}\right\rangle$, with $G_{1}=G_{2}$, we say that $M_{2}$ refines $M_{1}$ (or that $M_{1}$ is more abstract than $M_{2}$ ), denoted $M_{2} \preceq M_{1}$ iff $\mathcal{C}\left(M_{2}\right) \subseteq \mathcal{C}\left(M_{1}\right)$ over the same scope $S$.

E.g., the model $M_{e}^{H C 1}$ in Fig. 4(b) is more refined than $M_{e}$ in Fig. 1(g). In particular, $\mathcal{C}\left(M_{e}\right)$ consists of the models in Fig. 1(a)-(f), whereas $\mathcal{C}\left(M_{e}^{H C 1}\right)$ consists of the models in Fig. 1(a,b,e,f). Thus, the model $M_{e}^{H C 1}$ has less uncertainty.

A partial model without Maybe elements has exactly one concretization. The naming conventions in Sec. II allow us to define a unique conversion between a classical model and a corresponding partial model with a unique concretization.

Normal Forms. Given a set of classical models, there is no unique way to represent them as a partial model. For example, $M_{e}^{\neg H C 1}$ in Fig. 4(a) represents the models in Fig. 1(c, d). However, the same set of concretizations could be expressed by: (a) removing from the scope of $M_{e}^{\neg H C 1}$ extraneous False elements, such as the state Finishing, and (b) rewriting its propositional formula only in terms of its Maybe elements. In the case of $M_{e}^{\neg H C 1}$, the partial model has only one Maybe element (the transition on restart()), which can be either True or False, and therefore, the attached propositional formula $\phi_{e}^{\neg H C 1}$ is a tautology.

Definition 6: Two partial models $M_{1}, M_{2}$ are equivalent, denoted $M_{1} \sim M_{2}$, iff $\mathcal{C}\left(M_{1}\right)=\mathcal{C}\left(M_{2}\right)$. Obviously, $M_{1} \sim$ $M_{2}$ iff $M_{1} \preceq M_{2}$ and $M_{2} \preceq M_{1}$.

To help represent models, we define two normal forms: Graphical Normal Form (GNF) and Propositional Normal Form (PNF). Intuitively, a model in GNF represents most information in the graph, whereas in PNF it represents all the information in the formula. For example, the GNF and PNF for $M_{e}^{\neg H C 1}$ are shown in Fig. 3(a-b), respectively. In the latter, we did not represent False edges which would otherwise be represented by negated variables.

As partial models are complete graphs, the normal form of $M$ should be restricted to its largest complete subgraph that only contains True and Maybe nodes. We call the scope of this subgraph minimal. In the following, the symbol $\vDash$ signifies logical entailment.

Definition 7: Given a partial model $M=\langle G, \phi\rangle$, its GNF is a partial model $M^{\mathrm{GNF}}=\left\langle G^{\mathrm{GNF}}, \phi^{\mathrm{GNF}}\right\rangle$, constructed as follows:

- $G^{\mathrm{GNF}} \subseteq G$ and the scope $S$ of $M^{\mathrm{GNF}}$ is minimal.

- For every atom $a$ in $G$, if $\phi \vDash a$, then $a$ is annotated with True in $G^{\mathrm{GNF}}$.

- For every atom $a$ in $G$, if $\phi \vDash \neg a$, then $a$ is annotated with False in $G$ GNF.

- $\phi^{\mathrm{GNF}}$ is specified only in terms of elements annotated with Maybe in $G^{\mathrm{GNF}}$.

- $\phi \vDash \phi^{\mathrm{GNF}}$.

Proposition 1: Let $M$ be a partial model and $M^{\mathrm{GNF}}$ be a result of applying Definition 7. Then, $M \sim M^{\mathrm{GNF}}$.

Definition 8: Given a partial model $M=\langle G, \phi\rangle$, its PNF is a partial model $M^{\mathrm{PNF}}=\left\langle G^{\mathrm{PNF}}, \phi^{\mathrm{PNF}}\right\rangle$ constructed as follows:

- $G^{\mathrm{PNF}} \subseteq G$ and the scope $S$ of $M^{\mathrm{PNF}}$ is minimal.

- All elements in $G^{\mathrm{PNF}}$ are annotated with Maybe.

- $\phi^{\mathrm{PNF}} \vDash \phi$.

Proposition 2: Let $M$ be a partial model and $M^{\text {PNF }}$ be a result of applying Definition 8 . Then, $M \sim M^{\mathrm{PNF}}$.

Properties of Partial Models. The result of checking a property on a partial model can be True, False or Maybe. True means that the property holds for all concretizations, False that it does not hold for any of them, and Maybe that it holds for some, but not all concretizations. This is called thorough checking [2]. Moreover, by Definition 5, refinement preserves True and False properties. That is, as uncertainty gets reduced, values of properties about which we were certain remain unaffected.

\section{Reasoning with Partial Models}

In this section, we describe how to facilitate decision deferral in the presence of uncertainty by using partial models to reason with sets of alternatives. In particular, we define four reasoning operations:

OP1: Construction: how to create a partial model to (precisely) represent a set of alternatives.

OP2: Verification: how to check whether a partial model satisfies a property.

OP3: Diagnosis: how to find out which alternatives violate the property.

OP4: Refinement: how to filter out the alternatives that violate the property.

OP1: Construction. Construction of partial models is achieved by merging the alternatives and annotating the elements that vary between them by Maybe. Additionally, may formula is constructed to capture the allowable configurations of the Maybe elements.

Algorithm 1 shows how to create a partial model $M$ from a set $A$ of alternatives. By construction, $\mathcal{C}(M)=A$, which establishes the algorithm's correctness.

In our motivating example, the six alternative behavioral designs can be represented using the partial model shown 


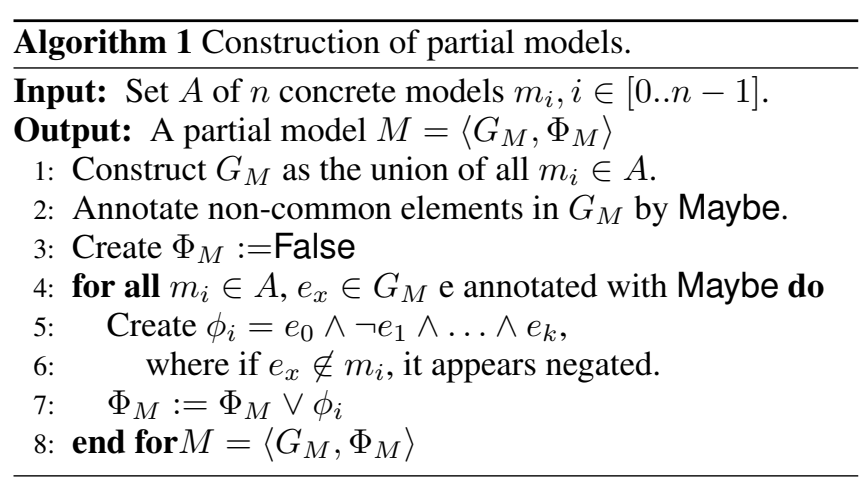

in Fig. 1(g). In the figure, elements annotated as Maybe appear dashed. For example, the state Finishing exists in only two alternatives, and the transition on restart () - in three; therefore, both are represented as Maybe. The rest of the elements are present in all of the alternatives, and thus are represented as True and appear solid. The corresponding may formula is shown in Fig. 1(g).

OP2: Verification. The purpose of the verification task is to answer the question "Does the desired property hold?".

In order to facilitate reasoning, we put the partial model in PNF and appropriately combine its PNF may formula with the formula representing the property we want to check. A SAT solver is then used to check whether the encoding of the model entails that of the property.

Specifically, the verification engine receives a partial model $M$ that is represented in PNF by the propositional formula $\Phi_{M}$ and a property expressed as a propositional formula $\Phi_{p}$. We then check satisfiability of the expressions $\Phi_{M} \wedge \neg \Phi_{p}$ and $\Phi_{M} \wedge \Phi_{p}$, using two queries to a SAT solver, combining the results to determine the outcome of the property on the partial model as described in Table I. For example, if both the property and its negation are satisfiable, then there is at least one concretization of the partial model where the property holds and another - where it does not. Thus, in the partial model the property has value Maybe.

Returning to our running example, in order to check whether the property $\mathrm{HC} 1$ holds for the partial model $M_{e}$ in Fig. $1(\mathrm{~g})$, we first put $M_{e}$ in PNF to get the propositional formula $\Phi_{e}$. Then we express $\mathrm{HC} 1$ as a propositional formula $\Phi_{H C 1}$, by grounding it over the vocabulary of $M_{e}$, as described in Sec. II. Checking the property means checking satisfiability $\Phi_{e} \wedge \neg \Phi_{H C 1}$ and $\Phi_{e} \wedge \Phi_{H C 1}$. The SAT solver returns one of the two models from Fig. 1(c, d) as the satisfying assignment for $\Phi \wedge \neg \Phi_{H C 1}$, and one of those in Fig. 1(a, b, e, f) for $\Phi \wedge \Phi_{H C 1}$. Thus, the value of HC1 is uncertain (Maybe) on the model.

OP3: Diagnosis. If the result of the verification task is False or Maybe, the next step is to do diagnosis, i.e., to answer the question "Why does the property of interest not hold?". Or, conversely, if the outcome was Maybe where it was expected to be False, to answer the question "Why is the property not violated?". Three forms of feedback can be returned:
Table I

CHECKING PROPERTY $p$ ON THE PARTIAL MODEL $M$.

\begin{tabular}{ccc}
$\Phi_{M} \wedge \Phi_{p}$ & $\Phi_{M} \wedge \neg \Phi_{p}$ & Property $p$ \\
\hline SAT & SAT & Maybe \\
SAT & UNSAT & True \\
UNSAT & SAT & False \\
UNSAT & UNSAT & (Inconsistent $M$ )
\end{tabular}

1) Return one counter-example - a particular concretization for which the property does not hold (OP3a): Such a counter-example is provided "for free" as a by-product of SAT-based verification. In particular, if the property is False, the SAT solver produces a satisfying assignment for $\Phi_{M} \wedge \neg \Phi_{p}$.

This assignment is a valuation for all propositional variables that correspond to elements in the scope of $M$ and can thus be visualized as a classical model for presentation to the user. To create the visualization, we conjoin all variables, negating those that had value False in the satisfying assignment. Provided the naming conventions in Sec. II are followed, this conjunction uniquely corresponds to a classical model, which is then presented as the feedback.

In our running example, verifying SC2 on the model $M_{e}$ involves checking the satisfiability of $\Phi_{e} \wedge \neg \Phi_{S C 2}$. This formula is satisfiable, and the SAT solver returns one of the concretizations in Fig. 1(e, f) as a satisfying assignment.

2) Return a concretization where the property does hold $(\boldsymbol{O P} 3 \boldsymbol{b})$ : This is also a by-product of the verification stage: if the result of checking the property is Maybe, the SAT solver produces a satisfying assignment for the formula $\Phi_{M} \wedge \Phi_{p}$. This valuation is expressed as a model (as discussed above) and provided to the user.

In the case of verifying SC2, the SAT solver returns a valuation that corresponds to one of the concretizations in Fig. 1(a,b,c,d) as a satisfying assignment to the formula $\Phi_{e} \wedge \Phi_{S C 2}$.

3) Return a partial model representing the set of all concretizations for which the property does not hold $(\boldsymbol{O P} 3 \boldsymbol{c})$ : These concretizations are characterized by the formula $\Phi_{M} \wedge$ $\neg \Phi_{p}$. In our example, the concretizations of $M_{e}$ that violate $\mathrm{HC} 1$ are those that satisfy the formula $\Phi_{e} \wedge \neg \Phi_{H C 1}$, i.e., those in Fig. 1(c,d).

In order to create useful feedback to the user, we consider a new partial model $M \neg p$ with the same vocabulary as $M$, that is represented in PNF by the formula $\Phi_{M} \wedge \neg \Phi_{p}$. We visualize $M \neg p$ by putting it into GNF. In our example, the partial model $M_{e}^{\neg H C 1}$ that represents the set of concretizations of $M_{e}$ that violate $\mathrm{HC} 1$ is shown in Fig. 4(a). $M_{e}^{\neg H C 1}$ is expressed in terms of the larger scope of $M_{e}$ and therefore certain elements are tagged as False and omitted from the diagram. The overall process is described in Algorithm 2. As the resulting model is constructed by the formula $\Phi_{M} \wedge \neg \Phi_{p}$, its set of concretizations is exactly the subset of concretizations of the original partial model for which the property was violated. In other words, $M \neg p \preceq M$.

OP4: Property-driven refinement. If the result of verification of an important property is Maybe, the developer may 


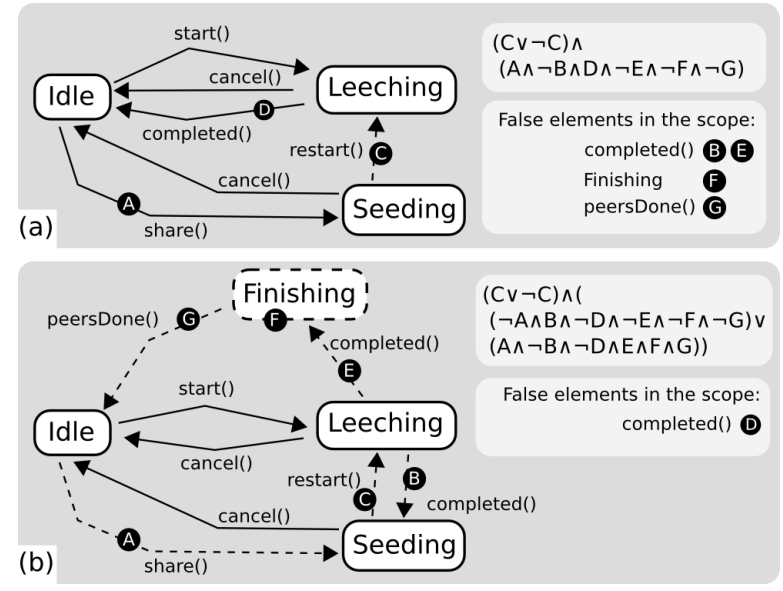

Figure 4. (a) Partial model $M_{e}^{\neg H C 1}$ representing all concretizations of $M_{e}$ that violate $\mathrm{HC} 1$. (b) Partial model $M_{e}^{H C 1}$ representing all concretizations of $M_{e}$ that satisfy $\mathrm{HC} 1$.

want to refine the partial model to a constrained version such that all of its concretizations satisfy the property. This subset of concretizations exactly characterized by the formula $\Phi_{M} \wedge \Phi_{p}$. We use it to construct the partial model $M^{p}$ in the same manner as we did for constructing $M \neg p$.

In our example, the set of concretizations of $M_{e}$ that satisfy $\mathrm{HC} 1$ consists of those in Fig. 1(a, b, e, f). Constructing the partial model $M_{e}^{H C 1}$ that represents these is done using the same method (shown in Algorithm 2) as for constructing its complement, $M_{e}^{\neg H C 1}$. Namely, the formula $\Phi_{e} \wedge \Phi_{H C 1}$ is constructed and then put into GNF. The result is shown in Fig. 4(b).

As $M^{p}$ is constructed using the formula $\Phi_{M} \wedge \Phi_{p}$, its set of concretizations is exactly the subset of concretizations of the original $M$ for which the property holds; thus, $M^{p} \preceq M$.

\section{EXPERIMENTS}

We conducted a preliminary empirical study to assess the feasibility and scalability of our approach to reasoning using partial models. More specifically, we attempted to answer the following research questions:

RQ1: How feasible is reasoning with sets of models with the partial model representation in comparison to the classical approach?

RQ2: How sensitive are the partial modeling representation and reasoning techniques to the varying degree of uncertainty?

To get answers to RQ1 and RQ2, we set up experiments with parameterized random inputs to simulate various categories of realistic reasoning settings.

Experimental setup. The reasoning tasks described in Sec. IV are operationalized using two fundamental tasks:

T1: Check the satisfiability of the formulas $\Phi_{M} \wedge \Phi_{P}$ and $\Phi_{M} \wedge \neg \Phi_{P}$ (for OP2, OP3a and OP3b).

T2: Construct a new partial model in GNF that has a PNF formula $\Phi_{M} \wedge \Phi_{P}$ (for OP3c with $\neg \Phi_{P}$ and OP4).

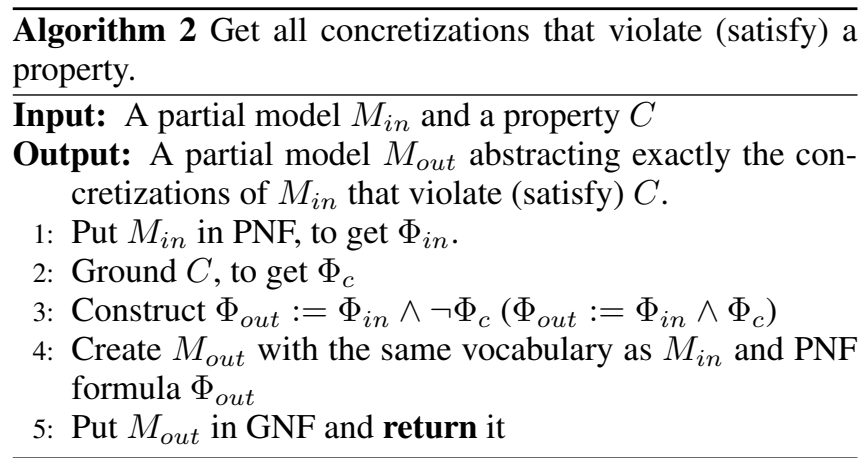

We focus our experimental evaluation on $\mathrm{T} 1$ and $\mathrm{T} 2$ because they require the use of SAT-solving technology, as opposed to Construction (OP1) which is linear to the number of input classical models and their elements (see Algorithm 1). Specifically, to answer RQ1, we conducted two experiments:

E1 Compare the relative performance of doing reasoning by running the task $\mathrm{T} 1$ to the performance of classical reasoning by considering the set of concretizations represented by $M$.

E2 Compare the relative performance of running T2 to get a partial model representing the subset of concretizations that satisfy a property, to the performance of incrementally collecting all the classical models as satisfying assignments of the formula $\Phi_{M} \wedge \Phi_{P}$.

To answer RQ2, we executed the experiments E1 and E2 with randomly generated experimental inputs that were parameterized to allow for different sizes, both with respect to model size and the size of the set of concretizations.

Experimental inputs. The metamodel of typed models corresponds to additional constraints in their propositional encoding. This makes the problem easier for the SAT solver, as it constrains the search space. We chose to use untyped models for inputs to our experiments, as the least constrained and thus the most difficult for the SAT solver.

We considered the following experimental parameters: 1) size of the partial model, 2) size of its set of concretizations, 3) quantification (e.g., existential, universal, mixed) of the property, and 4) result of property checking (True, False, Maybe). To manage the multitude of possible combinations of these, we discretized the domain of each parameter into categories.

We defined four size categories, based on the total number of elements (nodes and edges) in the partial model: Small (S), Medium (M), Large (L) and Extra-Large (XL). Based on pilot experiments, we defined ranges of reasonable values for each size category and selected a representative exemplar. The ranges of the categories and the selected exemplars for each category are shown in Table II.

In a similar manner, we defined four categories (S, M, L, $\mathrm{XL})$ for the size of the set of concretizations of the generated model. The size of this set reflects the degree of uncertainty encoded in the partial model, so that the category $\mathrm{S}$ corresponds to little uncertainty over which alternative to chose, 
Table II

CATEGORIES OF THE SIZE OF MODELS.

\begin{tabular}{|l|c|c|c|c|}
\hline Size of Model & S & M & L & XL \\
\hline \hline Nodes & $(0,10]$ & $(10,20]$ & $(20,40]$ & $(40,80]$ \\
\hline Elements & $(0,110]$ & $(110,420]$ & $(420,1640]$ & $(1640,6480]$ \\
\hline Exemplar & 30 & 240 & 930 & 2550 \\
\hline
\end{tabular}

Table III

CATEGORIES OF THE SIZE OF THE CONCRETIZATION SET.

\begin{tabular}{|l|c|c|c|c|}
\hline Size of Set & S & M & L & XL \\
\hline \hline Concretizations & $(0,10]$ & $(10,75]$ & $(75,150]$ & $(150,300]$ \\
\hline Exemplar & 5 & 50 & 100 & 200 \\
\hline
\end{tabular}

and the category XL corresponds to extreme uncertainty. Based on pilot experiments, we defined reasonable ranges and selected a representative exemplar for each category, as shown in Table III.

We also defined four property types (based on the quantification of FOL formulas): "fully existential" (E), "fully universal" (A) and two "mixed" categories: "exists-forall" (EA) and "forall-exists" (AE). Additionally, we considered the three possible results that can be yielded by property checking - True, Maybe and False.

Implementation. We implemented tooling support to randomly generate inputs based on the experimental properties outlined in Sec. V. Specifically, we generate propositional formulas expressed in the input format of the MathSAT 4 SMT Solver [3]. Each such propositional formula $\Phi_{r}$ is a conjunction of the form $\Phi_{r}=\Phi_{a} \wedge \Phi_{c} \wedge \Phi_{p}$, where $\Phi_{a}$ represents the annotations of the elements of the partial model, $\Phi_{c}$ - its set of concretizations and $\Phi_{p}$ - the property being checked. We describe these below.

For each random partial model, we considered a complete graph whose elements are in the model's finite vocabulary of $N_{1}$ nodes and $N_{1}^{2}$ edges. Each element is randomly annotated as True or False, and $N_{2}$ elements are annotated as Maybe. Each element in the model is represented by a boolean variable. The formula $\Phi_{a}$ captures the set of variables that make up the model as well as their annotations. In particular, $\Phi_{a}$ is a conjunction of $N_{1}\left(N_{1}+1\right)$ terms, one for each element. If an element $\alpha$ is annotated as True, its corresponding term is the non-negated variable $v_{\alpha}$. If it is annotated as False, its term is $\neg v_{\alpha}$, and if it is annotated as Maybe- $\left(v_{\alpha} \vee \neg v_{\alpha}\right)$. This tautological disjunction is necessary for $v_{\alpha}$ to be considered by the SAT solver even if it doesn't appear elsewhere in $\Phi_{r}$.

Each model is accompanied by the formula $\Phi_{c}$ that captures its set of concretizations. $\Phi_{c}$ is a disjunction of $N_{3}$ unique sub-formulas representing individual concretizations. Each one is a conjunction of the $N_{2}$ Maybe variables, a random number of which is negated. This way, each sub-formula defines an allowable configuration of Maybe elements.

Defining specific values for $N_{1}$ and $N_{3}$, we were able to generate models for each of the combinations of the parameters in Tables II and III.

To generate formulas $\Phi_{p}$ that simulate grounded FOL properties, we used property "templates". For example, to capture the FOL formula $\phi_{e x}=\exists x, y: x \Rightarrow y$, we created

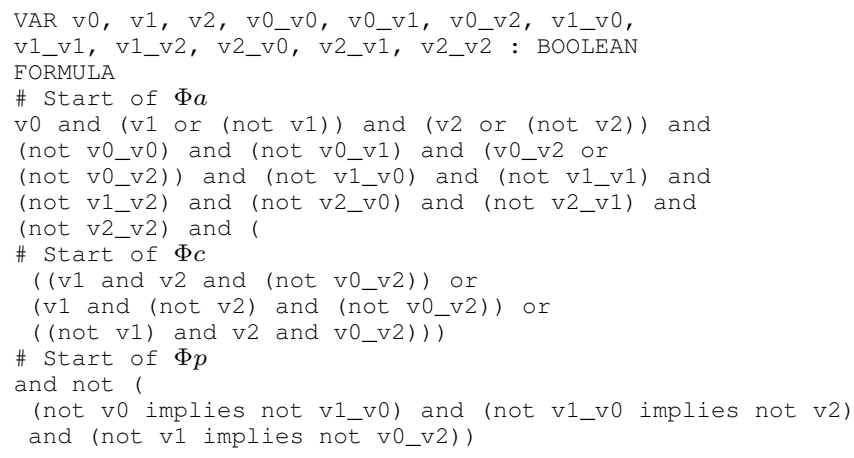

Figure 5. A randomly generated input in MathSAT's encoding language.

the template " $X$ implies $Y$ ". Given a partial model with elements represented by the set of variables $\left\{v_{1}, v_{2}, v_{3}, v_{4}\right\}$, the propositional formula that corresponds to grounding $\phi_{e x}$ over the vocabulary of the model is created as a randomly instantiated disjunction of copies of the template, e.g., "( $v 3$ implies v2) or (v1 implies v4)". To run experiments, our goal was creating templates for realistic properties such as the ownership relationship. E.g., the template "(not X) implies (not $Y$ )" indicates that $Y$ cannot exist without its "owner" element, $X$.

Each template was repeated $N_{6}$ times, with $N_{6}$ large enough so that $\Phi_{p}$ contains $N_{4}$ variables, out of which $N_{5}$ correspond to Maybe elements. Preliminary results by pilot experiments indicated that these parameters did not significantly affect the observed times and therefore in the generated inputs we fixed them to $N_{4}=0.1 \times N_{1}$ and $N_{5}=\min \left(N_{2}, 0.05 \times N_{1}\right)$.

To create properties in the FE ("fully existential") category, the template is repeated as a series of $N_{6}$ disjunctions and for FA properties - as a series of $N_{6}$ conjunctions. EA properties were generated as $N_{7}$ disjunctions of conjunctions of $N_{8}$ instantiations of the template, where $N_{7}$ and $N_{8}$ were random numbers s.t. $N_{7} \times N_{8}=N_{6}$. Similarly, AE properties were comprised of $N_{7}$ conjunctions of disjunctions of $N_{8}$ instantiations of the template.

Fig. 5 shows an example of an input formula generated randomly using a FA "ownership property" .

Methodology. We conducted a series of experiments generating inputs along the dimensions specified by three parameters: model size, size of set of concretizations and type of property. For each combination of the parameters, we produced inputs using the selected exemplar values shown in Tables II and III.We did multiple runs for each combination and then picked at least 3 runs for each data-point that produced results in each of the three possible return values (True, Maybe, False).

For each run, we used the generated input to execute the two experiments, E1 and E2. For each, we recorded the speedup $S_{p}=\frac{T_{c}}{T_{p m}}$, where $T_{c}$ and $T_{p m}$ were the times to do a task with sets of classical models and with partial models. 

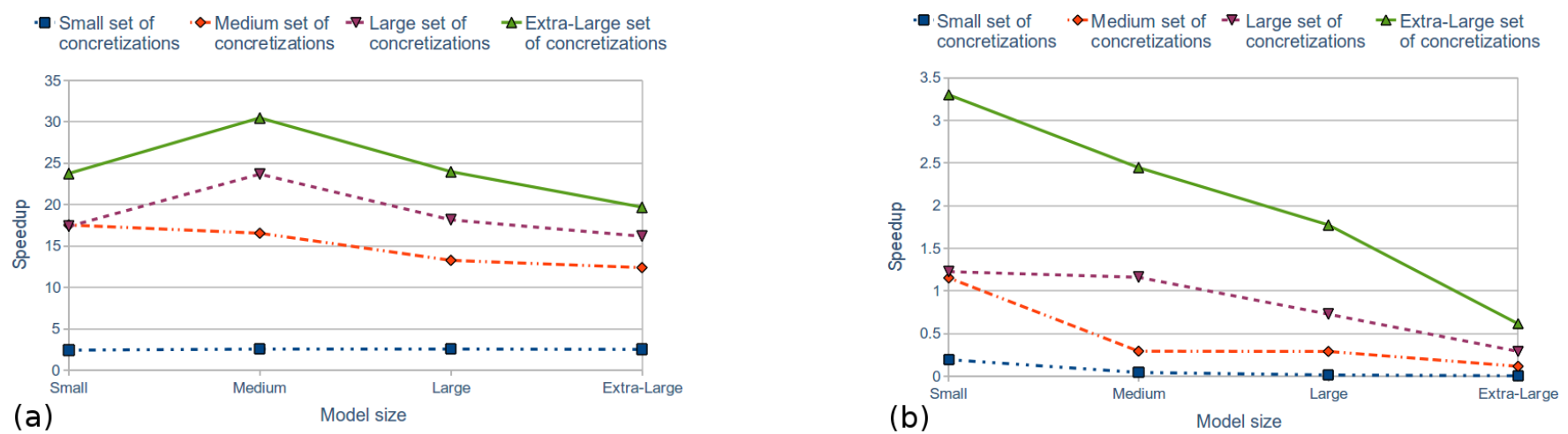

Figure 6. Speedup versus model size for different degrees of uncertainty: (a) experiment E1, (b) experiment E2.

Results. The experiments ${ }^{1}$ did not show dramatic differences in speedup between the different property and return types. The biggest difference in speedup for E1 was recorded in the $\mathrm{AE}$ category between properties that return Maybe (21.65) and those that return False (29.13), for M-sized models with L sets of concretizations. For E2, the biggest difference in speedup was recored for S-sized models with XL sets of concretizations, for properties that return Maybe, between the EA (0.36) and AE categories (5.62). This indicates that property and return types are not the prime determinants for the performance of our approach.

The size of the partial model and the size of the set of concretizations had a much larger effect on the recorded variance of speedup. The ranges of speedups for E1 and E2 are shown in Fig. 6(a, b), respectively. The plotted values are averages for type of property and return value for each combination of model size and size of set of concretizations. This indicates that these parameters are the most important factors for studying the effectiveness of partial model reasoning.

Fig. 6(a) shows that for verification and simple diagnostic tasks, such as producing a counter-example, there is a significant speedup from using partial models. The smallest speedups were observed in the inputs with $S$ sets of concretizations (between 2.45 for S-sized models and 2.59 for Lsized models). The increase from these values was dramatic for M, L and XL sets of concretizations. For these categories, the smallest speedup was 19.72 for XL-sized models with M sets of concretizations and the biggest speedup was 30.49 for M-sized models with XL sets of concretizations.

For more complex tasks, such as property-driven refinement, the effect of the size of concretizations, as shown in Fig. 6(b), seems to be the determinant parameter, as the technique offers a speedup greater than 1 for larger sets of concretizations. Our approach was significantly slow for $\mathrm{S}$ sets of concretizations (the largest speedup being 0.05 for Msized models). Moderate speedups were only recorded for XL sets of concretizations and smaller models (3.30 for S, 2.25 for $\mathrm{M}$ and 1.78 for $\mathrm{L}$ ). This points to the conclusion that for more complex tasks, speedup is best for smaller models with larger sets of concretizations.

\footnotetext{
${ }^{1}$ All results available at http://www.cs.toronto.edu/ famelis/icse $12 . h t m l$
}

These observations, lead us to the conclusion that, regarding RQ1 (feasibility), there is a significant net gain from using our approach for tasks like verification and counterexample guided diagnosis, whereas for tasks like propertydriven refinement there are certain cases where it is preferable to use the classical approach.

Regarding RQ2 (sensitivity to degree of uncertainty), the observations point to the conclusion that the speedup offered by our approach is positively correlated to the degree of uncertainty. In fact, the greatest speedups were observed for inputs that had bigger sizes of sets of concretizations. For smaller levels of uncertainty, explicitly handling the set is more efficient.

Threats to Validity. The most important threat to validity stems from the use of experimental inputs that were randomly generated. The formulas that we created for properties were randomly grounded and were generated from a few arbitrarily defined templates.

Another threat to validity is induced by our choice to use a few exemplar values of the experimental parameters in order to manage the combinatorial explosion of options. It is evident that more experimentation is required, to generalize our results and further investigate effects of the experimental parameters that may not have been made obvious by our set of experiments.

To compensate for these threats to validity, we additionally conducted a Case Study, to triangulate our experimental results with experience from applying our technique to a real world application. The size of the models that we extracted from the Case Study fell in the XL category, with M and L sets of concretizations, whereas the properties were in the FE category and returned True and Maybe. The observed speedups (detailed in the next section) )were consistent with our experimental results.

\section{CAse Study}

Problem Description. In this case study, we aim to illustrate the following MDE software maintenance scenario: An engineer is given the task of fixing a software defect by modifying its UML model which will subsequently be used to construct the modified software (e.g., via a transformation). However, 
after creating the modifications to the model, the engineer finds that some model constraints are violated and thus the software cannot be constructed. For example, she may have modified a sequence diagram without properly synchronizing it with the structural aspects (e.g., classes) of the model. To help her resolve these constraint violations, she uses a tool that can automatically propose different model repair alternatives (e.g., [12]). Suppose the engineer is uncertain about which alternative to choose because their relative merits are unclear - and thus she would like to reason with the set of alternatives to help her make the choice and possibly even defer the decision until more information is available. In this case study, we apply the partiality techniques developed in this paper to show how they could help her in this scenario and to illustrate the feasibility of the approach.

We use an open source project UMLet [23], which is a simple Java-based UML editor, as the software on which our user is requested to perform a maintenance task. This project has also been used by Van Der Straeten et al. for finding model inconsistencies with a model finder [24]. The goal of the maintenance task is to fix the following bug, referred to as Issue 10 on the online issue log [22]: "copied items should have a higher z-order priority". That is, if the user copies and then pastes an item within the editor, it is not the topmost item if it overlaps with other existing items. Thus, any fix to the bug must satisfy the following property P1: "Each item that is pasted from the clipboard must have z-order $=0$." The paste functionality is implemented in UMLet by instantiating the class Paste and invoking its execute operation. Fig. 7 shows a fragment of the sequence diagram, generated from the code using the Borland TogetherJ tool [1] for execute with the circled portion representing a bug fix we propose. The full sequence diagram has 12 objects, 53 messages and 8 statement blocks. Although UMLet has 214 classes in total, we restrict ourselves to a slice that covers the sequence diagram for execute consisting of 6 classes (plus 5 Java library classes) with 44 operations. Of the 12 objects in the sequence diagram, 5 are instances of Java library classes and $7-$ of UMLet classes. In the fragment shown, the for loop statement block iterates through every item in the clipboard (indexed by variable $e$ ) and adds it to the editor window (represented by the object pnl:DrawPanel). When an entity is added to a DrawPanel, the z-order is not set to 0 by default, causing the bug. In our proposed fix (shown in the dashed circle), we create a transient object positioner and tell it to moveToTop $(e)$, using the Swing operation setComponentZOrder.

Inconsistencies and the Partial Model. Our fix is conceptually correct but it violates two consistency rules required for code generation:

1) ClasslessInstance: Every object must have a class. Possible repairs:

- RC1: Remove the object.

- RC2:(obj) Replace the object with an existing object obj that has a class.

- RC3:(cls) Assign the object to the existing class $c l s$.

- RC4: Assign the object to a new class.

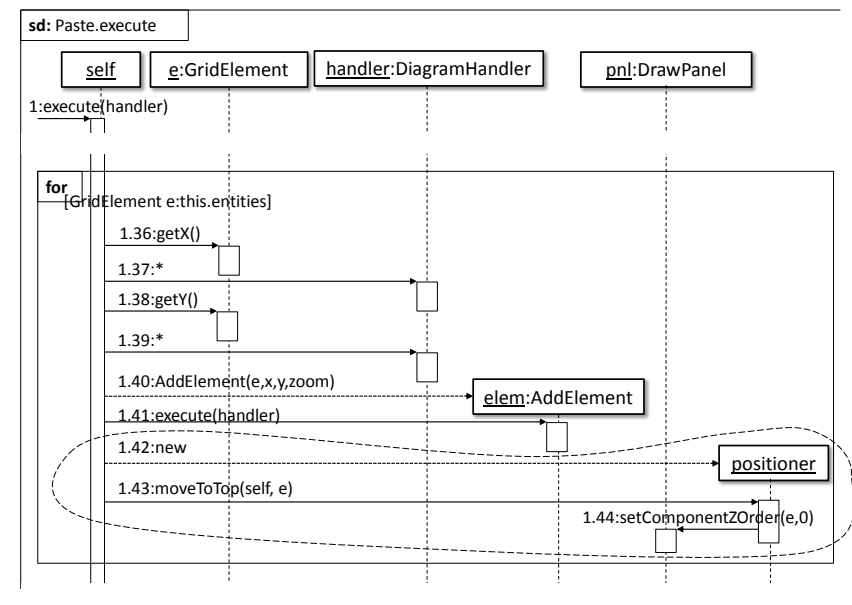

Figure 7. Our fix for the execute operation of the UMLet paste function.

2) DanglingOperation: The operation used by a message in a sequence diagram must be an operation of the class of the receiving object. Possible repairs:

- RD1: Put the operation into the receiving object's class.

- RD2:(op) Change the operation to the operation $o p$ that is already in the receiving object's class.

- RD3: Remove the message.

ClasslessInstance and DanglingOperation are both based on [21]. In our case, the positioner object violates ClasslessInstance and the message with operation moveToTop violates DanglingOperation because it is not in positioner's class (since positioner has no class).

If we apply all possible repairs, we get a set of alternative ways to fix the inconsistency, summarized as follows:

1) Positioner can be removed $(R C 1)$, can be replaced by one of the existing 7 objects $(R C 2)$, can be assigned to one of the existing 6 classes (RC3), or can be an instance of a new class (RC4).

2) The operation moveToTop can be added to the positioner's class $(R D I)$, can be changed to one of the other 44 operations depending on positioner's class (RD2), or can be removed (RD3).

Only certain repairs are mutually compatible - for example, $R C 1$ cannot be used with $R D 2$ since the latter depends on positioner's class but the former removes positioner entirely. There are 220 alternatives in total for all valid combinations.

If we construct a partial model to represent this set of alternatives, all the model elements in the proposed fix in Fig. 7 become Maybe since they are present in some alternatives and absent in others. Furthermore, based on the compatible combinations of repairs, the may formula portion of the partial model is expressed as

$$
\begin{aligned}
\phi_{M}= & \text { Choose }\left(\left(\phi_{R C 1} \wedge \phi_{R D 3}\right),\right. \\
& \left.\left(\phi_{R C 2(e)} \wedge \phi_{R D 1}\right),\left(\phi_{R C 2(e)} \wedge \phi_{R D 2(\text { get } X)}\right), \ldots\right),
\end{aligned}
$$

where Choose $\left(\phi_{1}, \ldots, \phi_{n}\right)$ is a logical function that holds when exactly one of $\left\{\phi_{i}\right\}_{1 \leq i \leq n}$ hold. Each of the formulas 
for the individual repairs can be further expanded and expressed in terms of the UML 2 metamodel [16]. For example, $\phi_{R C 2(e)}$ represents the condition that object positioner is replaced by object $e$ in Fig. 7, expressed as

$$
\phi_{R C 2(e)}=\operatorname{covered}(\text { receiveEvent }(\text { Message_1.43) })=\text { Lifeline_e }
$$

which says that the lifeline covered by the receiving event of message 1.43 is the one for object $e$ :GridElement.

Analysis. Having defined a partial model whose set of concretizations are the possible alternative ways of making our bug fix consistent with the required rules, we can use the techniques discussed in Sec. IV to reason about the alternatives using properties. The first question is whether any of the alternatives "break" the paste functionality. For example, consider the property P2: "Whenever an item is pasted, a new item is created in the editor window" which should hold if the paste functionality is implemented correctly. To check this against the partial model, we encode it into a propositional formula $\phi_{P 2}$ over the UML metamodel in the same way as the repair formulas. Due to lack of space, we limit ourselves to a high-level description of the encoding: " $\phi_{P 2}$ holds iff in the sequence diagram for execute there exists an iteration over the items in the clipboard (e.g., a for block) that creates a copy of each item using the operation CloneFromMe() and later adds the item to the editor window using the command AddElement". Only the AddElement portion is visible in the fragment in Fig. 7.

We used the MathSAT implementation described in Sec. V to verify this property (OP2), checking the queries $\phi_{M} \wedge \phi_{P 2}$ and $\phi_{M} \wedge \neg \phi_{P 2}$. The result was True, indicating that all concretizations satisfy the property. We also did the comparison with doing this task using classical models as in Sec. V and found a speedup of 30.68. Next we considered the critical property $\mathrm{P} 1$ that is required for any fix to our bug. In this case, OP2 yielded Maybe indicating that some but not all concretizations are acceptable bug fixes. In this case, we found a speedup of 31.23 .

To investigate why some concretizations did not fix the bug, we used our diagnosis technique (OP3c) to produce the partial model representing the counterexamples to P1 by setting the may formula to $\phi_{M} \wedge \neg \phi_{P 1}$ and computing the GNF. In the resulting may model, the moveToTop operation is absent and thus moveToTop is necessary for P1 to hold. This is reasonable since if this operation is not invoked, then the z-order is never set to 0. In a similar way, we used property-guided refinement (OP4) to refine our partial model to represent only the satisfying concretizations by setting the may formula $\phi_{M} \wedge \phi_{P 2}$ and computing the GNF. The speedup for these two tests were 0.19 and 0.05 , respectively.

\section{RELATED WORK}

A number of partial behavioral modeling formalisms, have been studied in the context of abstraction (for verification) or for capturing early design models [9]. For example, Modal Transition Systems (MTSs) [10] allow introduction of uncertainty about transitions on a given event, whereas Disjunctive Modal Transition Systems (DMTSs) [11] add an additional constraint that at least one of the possible transitions must be taken in the refinement. These approaches compactly encode an over-approximation of the set of possible LTSs and thus reasoning over them suffers from information loss. Moreover, the MTS and DMTS refinement mechanism allows resulting LTS models to have an arbitrary number of states which is different from the treatment provided in this paper, where we concentrated only on "structural" partiality and thus state duplication was not applicable.

Another relevant area is product line software development [17] which captures the set of potential models by identifying their commonalities and variabilities. Most approaches keep the expressions of variability in a separate feature model but some incorporate these directly into the model using notational extensions in the metamodel [13]. Featured Transition Systems (FTSs) [4] are most closely related to the notion of partial models presented in this paper. FTSs encode a set of products by annotating transitions with specific features from a feature diagram (much like our may formula), and differ from MTSs and DMTSs in that they support precise representation and reasoning with a set of models.

Our approach is distinct from related work in a number of important ways. First, it applies to any kind of modeling language (not just behavioral models) that can be defined using a metamodel. Second, our viewpoint is the comprehensive handling of uncertainty rather than just reasoning over variability. In this context, partial models support changes in the level of uncertainty, with tasks such as property-driven (OP4) and more generally uncertainty-removing refinement [19]. Third, partial models are first-class development artifacts that can be manipulated throughout the software engineering life cycle with detail-adding transformations that do not affect the level of uncertainty [8]. Finally, the notion of partiality studied here (where model elements can be optional or mandatory) is only one of several kinds of partiality, developed in [20].

\section{CONCLUSION AND FUtURE WORK}

This paper presented an approach for reasoning in the presence of uncertainty. We showed how to construct partial models to represent sets of alternatives and how to use them for reasoning. We evaluated the approach by running experiments using randomly generated inputs and triangulated our results with a case study dealing with alternative repairs to inconsistency for a real world software project. Our evaluation, while preliminary, showed that in the presence of high degrees of uncertainty, using partial models offers significant improvements for reasoning tasks.

Our work is part of a broader research agenda, outlined in [7]. Our next steps include studying how partial models can be used as first-class development items. In particular, we want to investigate model transformation of partial models, as well as the effects of transformation on the properties of the concretizations. 


\section{REFERENCES}

[1] Borland TogetherJ. website : http://www.borland.com/us/products/together/, accessed 0320-2012.

[2] G. Bruns and P. Godefroid. "Model Checking Partial State Spaces with 3-Valued Temporal Logics". In Proc. of CAV'99, pages 274-287, 1999.

[3] R. Bruttomesso, A. Cimatti, A. Franzen, A. Griggio, and R. Sebastiani. "The MathSAT 4 SMT Solver". In Proc. of CAV'08, pages 299-303, 2008.

[4] P. Classen, A.and Heymans, P. Schobbens, A. Legay, and J. Raskin. "Model Checking Lots of Systems: Efficient Verification of Temporal Properties in Software Product Lines". In Proc. of ICSE'10, pages 335-344, 2010.

[5] A. Egyed, E. Letier, and A. Finkelstein. "Generating and Evaluating Choices for Fixing Inconsistencies in UML Design Models". In Proc. of ASE'08, pages 99-108, 2008.

[6] H. Ehrig, K. Ehrig, U. Prange, and G. Taentzer. Fundamentals of Algebraic Graph Transformation. EATCS. Springer, 2006.

[7] M. Famelis, S. Ben-David, M. Chechik, and R. Salay. "Partial Models: A Position Paper". In Proc. of MoDeVVa'11, pages $1-6,2011$.

[8] M. Famelis, R. Salay, and M. Chechik. "The Semantics of Partial Model Transformations”. In Proc. of MiSE'12, 2012. To appear.

[9] D. Fischbein, G. Brunet, N. D'ippolito, M. Chechik, and S. Uchitel. "Weak Alphabet Merging of Partial Behaviour Models". ACM TOSEM, 21, 2012.

[10] K. G. Larsen and B. Thomsen. "A Modal Process Logic". In Proc. of LICS'88, pages 203-210, 1988.

[11] P. Larsen. "The Expressive Power of Implicit Specifications". In Proc. of ICALP'91, volume 510 of $L N C S$, pages 204-216, 1991.

[12] T. Mens and R. V. D. Straeten. "Incremental Resolution of Model Inconsistencies". In Proc. of WADT'06, 2007.

[13] B. Morin, G. Perrouin, P. Lahire, O. Barais, G. Vanwormhoudt, and J. M. Jézéquel. "Weaving Variability into Domain Metamodels". J. Model Driven Engineering Languages and Systems, pages 690-705, 2009.
[14] C. Nentwich, W. Emmerich, and A. Finkelstein. "Consistency Management with Repair Actions". In Proc. of ICSE'03, pages 455-464, 2003.

[15] Object Management Group. Object Constraint Language OMG Available Specification Version 2.0, 2006.

[16] OMG. UML Superstructure Specification Version 2.3, 2010

[17] K. Pohl, G. Böckle, and F. Van Der Linden. Software Product Line Engineering: Foundations, Principles, and Techniques. Springer-Verlag New York Inc, 2005.

[18] M. Sabetzadeh, S. Nejati, M. Chechik, and S. Easterbrook. "Reasoning about Consistency in Model Merging". In Proc. of LWI'10, 2010.

[19] R. Salay, M. Chechik, and J. Gorzny. "Towards a Methodology for Verifying Partial Model Refinements". In Proc. of VOLT'12, 2012. To appear.

[20] R. Salay, M. Famelis, and M. Chechik. "Language Independent Refinement using Partial Modeling”. In Proc. of FASE'12, 2012.

[21] R. V. D. Straeten, T. Mens, J. Simmonds, and V. Jonckers "Using Description Logic to Maintain Consistency between UML Models". In Proc. of UML'03, pages 326-340, 2003.

[22] UMLet. UMLet Issue List : http://code.google.com/p/umlet/issues/list, accessed 03-202012.

[23] UMLet. UMLet website : http://www.umlet.com/, accessed 0320-2012.

[24] R. Van Der Straeten, J. Pinna Puissant, and T. Mens. "Assessing the Kodkod Model Finder for Resolving Model Inconsistencies". Modelling Foundations and Applications, 6698:6984, 2011.

[25] A. Van Deursen, P. Klint, and J. Visser. "Domain-Specific Languages: An Annotated Bibliography". ACM Sigplan Notices, 35(6):26-36, 2000.

[26] A. van Lamsweerde. Requirements Engineering - From System Goals to UML Models to Software Specifications. Wiley, 2009.

[27] H. Ziv, D. Richardson, and R. Klösch. "The Uncertainty Principle in Software Engineering”, 1996. 